\title{
Sequential coalition formation and the core in the presence of externalities
}

Citation for published version (APA):

Kóczy, L. Á. (2006). Sequential coalition formation and the core in the presence of externalities. METEOR, Maastricht University School of Business and Economics. METEOR Research Memorandum No. 047 https://doi.org/10.26481/umamet.2006047

Document status and date:

Published: 01/01/2006

DOI:

10.26481/umamet.2006047

Document Version:

Publisher's PDF, also known as Version of record

\section{Please check the document version of this publication:}

- A submitted manuscript is the version of the article upon submission and before peer-review. There can be important differences between the submitted version and the official published version of record.

People interested in the research are advised to contact the author for the final version of the publication, or visit the DOI to the publisher's website.

- The final author version and the galley proof are versions of the publication after peer review.

- The final published version features the final layout of the paper including the volume, issue and page numbers.

Link to publication

\footnotetext{
General rights rights.

- You may freely distribute the URL identifying the publication in the public portal. please follow below link for the End User Agreement:

www.umlib.nl/taverne-license

Take down policy

If you believe that this document breaches copyright please contact us at:

repository@maastrichtuniversity.nl

providing details and we will investigate your claim.
}

Copyright and moral rights for the publications made accessible in the public portal are retained by the authors and/or other copyright owners and it is a condition of accessing publications that users recognise and abide by the legal requirements associated with these

- Users may download and print one copy of any publication from the public portal for the purpose of private study or research.

- You may not further distribute the material or use it for any profit-making activity or commercial gain

If the publication is distributed under the terms of Article $25 \mathrm{fa}$ of the Dutch Copyright Act, indicated by the "Taverne" license above, 
László Á. Kóczy

Sequential Coalition Formation and the Core in the Presence of Externalities

RM/06/047

JEL code : C71, C72

\section{METE@R}

Maastricht research school of Economics of TEchnology and ORganizations

Universiteit Maastricht

Faculty of Economics and Business Administration P.O. Box 616

NL - 6200 MD Maastricht

phone : ++31433883830

fax : ++31433884873 



\title{
SEQUENTIAL COALITION FORMATION AND THE CORE IN THE PRESENCE OF EXTERNALITIES
}

\author{
LÁSZLÓ Á. KÓCZY
}

\begin{abstract}
Bloch (1996) presents a novel method to solve cooperative games with externalities. When trying to relate this to classical approaches he could only present negative results. We elaborate on these problems, define a modification of Bloch's model and show that its order-independent equilibria coincide with the (pessimistic) recursive core (Kóczy, 2005).

Subject classification: $\mathrm{C} 71, \mathrm{C} 72$

Keywords and phrases: Core, externalities, sequential coalition formation, order-independent equilibria
\end{abstract}

\section{INTRODUCTION}

Solving cooperative games with externalities remains an interesting, important but difficult problem in game theory. While for games without externalities, a plethora of concepts have been proposed it proved to be difficult to generalise these solutions, and often the games were simplified to fit old models instead.

The sequential model of coalition formation (Bloch, 1996) is certainly one of the first models that is attractive due to its simplicity, but also, since this is a noncooperative model, as it does not only give the equilibrium coalition structures, but also some insight why precisely these form. (For an application see Bloch, 1995)

One of the few drawbacks of the model was that despite the apparent logical relation to standard cooperative concepts, in particular, the core, a clear relation could not be established. In fact, (Bloch, 1996) presents an example proving the concept to be unrelated to the $\alpha$-core, the closest suspected relative.

In this paper we reconsider this issue and explain the reasons for this negative result. We also show that Bloch's model would for some games result in Paretodominated equilibrium coalition structures. We present a modification that is free from these deficiencies. We show not only a well-defined relation to the $\alpha$-core, but show that, when all residual cores are nonempty, the pessimistic recursive core (Kóczy, 2005) coincides with set of order-independent equilibrium coalition structures as defined by the modified model.

In recursive cores a deviation by a set of players is responded by an equilibrium partition of the residual players. The equilibrium is nothing but the solution of the restricted game induced by the deviation. While optimism or pessimism of these players plays a role here, too, as this model takes the behaviour of the residual players explicitly into account, it gives a better prediction of the possible responses, of the profitability of deviations and ultimately of the stability of a particular

The author thanks Francis Bloch, Luc Lauwers and Geert Dhaene for discussions and encouragement; and acknowledges the support of the Netherlands Organisation for Scientific Research and of the European Union (MEIF-CT-2004-011537). 
partition. Huang and Sjöström (2003) have independently formulated a similar model, the $r$-theory for normal form games.

Our paper is a contiunation of the literature studying the relation of the core and noncooperative coalition formation (Chatterjee et al., 1993; Lagunoff, 1994; Perry and Reny, 1994), but while these papers deal with characteristic function form games we allow for externalities. It extends the results of Moldovanu and Winter (1995) to discrete partition function form games (Lucas and Macelli, 1978). Our work is most related to Huang and Sjöström (2006) who independently established a similar result modifying the model of Perry and Reny (1994). As in this model the order of players is not fixed, order independence is not an issue, their focus is on stationary subgame perfect equilibria and show equivalence to the r-core (Huang and Sjöström, 2003) for totally r-balanced games (a condition that resembles our assumption of non-empty residual cores).

The structure of the paper is as follows. After the introduction of Bloch's model, we explain the two aforementioned objections. The third section contains our modified model. Then we present the recursive core and finally our main results.

\section{Preliminaries}

Let $N$ be a finite set of players. Subsets of $N$ are called coalitions. A partition $\pi$ of $N$ is a breaking up of $N$ into disjoint coalitions. $\Pi_{S}$ is the set of partitions of any set $S$ of the players with $\pi_{S}$ denoting a typical element. The game is also featured by a discrete partition function (Lucas and Macelli, 1978) $v: \Pi(N) \longmapsto \mathbb{R}^{N}$. The component $v_{i}(\pi)$ denotes the payoff for player $i$ in case partition $\pi$ formed. For vectors $x, y \in \mathbb{R}^{N}$ we write $x>_{S} y$ if $x_{i} \geq y_{i}$ for all $i \in S \subset N$ and there exists $j \in S$ such that $x_{j}>y_{j}$.

A rule of order $\rho$ is an ordering of the players that determines the order in which players make their moves in the sequential game of coalition formation. Let $\rho(S)$ denote the player ranked first among the members of $S$. For instance, $\rho(N)$ is the player starting the game. A game of sequential coalition formation or simply a game will be fully described by the discrete partition function and the rule of order and hence is denoted as $\Gamma(v, \rho)$.

The game shall be played as follows.

(1) We start the game at the first player.

(2) The current player makes a proposal. A proposal is a coalition of players.

(3) The following player in this coalition gets the word. He can either reject the proposal, become the next proposer and the game continues at step 2 . Alternatively he can accept the proposal and the step is repeated.

When a proposal is made the following player is the highest ranked player who has not spoken yet: in case the last player made a proposal, then this is the highest ranked player in the coalition (apart from the proposer), otherwise it is the player who follows in rank.

A history $h^{t}=\left(\hat{K}\left(h^{t}\right), \pi_{\hat{K}\left(h^{t}\right)}, \hat{T}\left(h^{t}\right), S, i\right)$ at date $t$ is a list of offers, acceptances and rejections up to period $t$. The history therefore determines

- a set $\hat{K}\left(h^{t}\right)$ of players who have already formed coalitions and left the game,

- the coalition structure $\pi_{\hat{K}\left(h^{t}\right)}$ of players in $\hat{K}\left(h^{t}\right)$,

- a -possibly empty- ongoing proposal $\hat{T}\left(h^{t}\right)$,

- a set of players $S$ who have already accepted the proposal, 
- a player $i$ who moves at time $t$.

A player $i$ is active at history $h^{t}$ if it is his turn to move. The collection of histories at which $i$ is active is denoted $H_{i}$. A strategy $\sigma_{i}$ for player $i$ is a mapping from $H_{i}$ to his set of actions:

$$
\sigma_{i}\left(h^{t}\right) \in \begin{cases}\{\text { Yes, No }\} & \text { if } \hat{T}\left(h^{t}\right) \neq \emptyset \\ \mathcal{T}\left(i, \hat{K}\left(h^{t}\right)\right) & \text { if } \hat{T}\left(h^{t}\right)=\emptyset\end{cases}
$$

where $\mathcal{T}\left(i, \hat{K}\left(h^{t}\right)\right)=\left\{T \subseteq N \backslash \hat{K}\left(h^{t}\right), i \in T\right\}$, the set of coalitions that $i$ can form with the remaining set of players. See also Bloch (1996).

A strategy profile uniquely determines an outcome $(\pi(\sigma), t(\sigma))$ of the game. In case $t(\sigma)$ is finite, $\pi(\sigma)$ is a partition of the set $N$, otherwise of a strict subset of $N$. In the latter case players who could not agree on the coalition to form are offered a payoff that is less than the payoff they would receive in any partition. In this case the payoff of the players who have already left the game is not clear: Bloch (1996) chose to take the most favourable case for the players: $v_{i}(\pi(\sigma))=\max _{\pi_{K} \subset \pi} v_{i}(\pi)$.

We are interested in stationary strategies:

Definition 1. A strategy is stationary if it does not depend on the history, but only on the current state $s=\left(K, \pi_{K}, T\right)$.

Definition 2. A subgame-perfect equilibrium $\sigma^{*}$ is a strategy profile such that for all players $i \in N$, for all histories $h^{t} \in H_{i}$ and for all strategies $\sigma_{i}$ of player $i$ we have

$$
v_{i}\left(\pi\left(\sigma_{i}^{*}\left(h^{t}\right), \sigma_{-i}^{*}\right)\right) \geq v_{i}\left(\pi\left(\sigma_{i}\left(h^{t}\right), \sigma_{-i}^{*}\right)\right)
$$

Definition 3. A stationary perfect equilibrium is an subgame-perfect equilibrium profile that is also stationary.

2.1. Two negative results. Bloch (1996) shows that for each game $\Gamma(v, \rho)$ there exists a subgame-perfect equilibrium, however stationary perfect equilibria may fail to exist. He then focuses on outcomes of stationary perfect equilibria (in finite time) and attempts to establish a link to existing cooperative solution concepts. After some encouraging results Bloch (1996, pp 105-106) presents an example that produces a non-empty set of stationary equilibrium coalition structures (SECS), a nonempty $\alpha$-core, but the intersection of the two solutions is empty suggesting that there is no relation between the two concepts.

In the following section we make a small modification to the sequential coalition formation game that makes a stronger relation possible.

Consider also the following example. It is an example based on the Cournot oligopoly game studied by Bloch (1996, Section 5.1) with a slight difference: No majority coalitions are permitted to form. Such a game can easily emerge as a result of antitrust regulations. We model this by assigning very low payoffs for players in majority coalitions.

Example 1. In a Cournot oligopoly game with a linear inverse demand function $D=\alpha-Q$ and uniform marginal cost $\lambda$ a player belonging to a cartel of size $t(i)$ active in a market of $K$ cartels gets a payoff of $\frac{(\alpha-\lambda)^{2}}{t(i)(K+1)^{2}}$. Consider a game with 4 players. The game is symmetric, and therefore any choice of $\rho$ is suitable. The payoffs (up to the constant multiplier $(\alpha-\lambda)^{2}$ ) are as follows: 


\begin{tabular}{l|cccc}
$\pi$ & $v_{i}$ & $v_{j}$ & $v_{k}$ & $v_{l}$ \\
\hline$\{i, j, k, l\}$ & $\frac{1}{25}$ & $\frac{1}{25}$ & $\frac{1}{25}$ & $\frac{1}{25}$ \\
$\{i j, k, l\}$ & $\frac{1}{32}$ & $\frac{1}{32}$ & $\frac{1}{16}$ & $\frac{1}{16}$ \\
$\{i j, k l\}$ & $\frac{1}{18}$ & $\frac{1}{18}$ & $\frac{1}{18}$ & $\frac{1}{18}$ \\
any other & 0 & 0 & 0 & 0
\end{tabular}

where e.g. $i j$ stands for a coalition consisting of players $i$ and $j$ and $i, j, k, l$ are permutations of $1,2,3,4$.

Lemma 1. The partition $\{i, j, k, l\}=\{1,2,3,4\}$ is the unique stationary equilibrium coalition structure of this game.

Proof. The proof is a straightforward solution of the game.

When a single player is left over, it forms a singleton coalition.

Now assume that $K=\{i, j\}$ have left the game. W.l.o.g. $k$ is the active player. Two cases are considered:

$\pi_{K}=\{i, j\}$ : Proposing a singleton pays $\frac{1}{25}$, becoming a pair pays $\frac{1}{32}$ : such a proposal would not be made nor accepted.

$\pi_{K}=\{i j\}$ : Proposing a singleton pays $\frac{1}{16}$, becoming a pair pays $\frac{1}{18}$ : again, such a proposal would not be made nor accepted.

When only a single player has left the game, proposing a triple is always dominated: e.g. going single would play at least $\frac{1}{16}>0$. For the other proposals we already know the coalition structure that the remaining players will form and proposing a singleton is the dominant strategy.

Finally we consider the case when all 4 players are still in the game. Proposing a 3 or 4-player coalition is dominated as before. Since the remaining players will become singletons, proposing or accepting a pair will result in a payoff of $\frac{1}{32}$, proposing a singleton yields $\frac{1}{25}$, hence proposing a singleton is yet again the equilibrium strategy.

Therefore, in equilibrium, the all-singletons coalition structure will form.

It is clear that the coalition structure $\{i j, k l\}$ is preferred to $\{i, j, k, l\}$ by all players.

Corollary 2. Stationary equilibrium coalition structures can be Pareto-dominated by coalition structures that cannot be produced by stationary equilibria.

Note that since here the $\alpha$-core consists of the coalition structure $\{i j, k l\}$, our example is also an example of a game where $\operatorname{SECS}(v, \rho) \neq \emptyset$ and $C \alpha(v) \neq \emptyset$, but $\operatorname{SECS}(v, \rho) \cap C \alpha(v)=\emptyset$.

\section{MODIFIED SEQUENTIAL COALITION FORMATION GAME}

When introducing the $\alpha$-core Bloch (1996) writes: "In the $\alpha$ definition, a group $K$ of players deviates if there exists a coalition structure $\pi_{K}$ such that, whatever the reaction of the external players, members of $K$ are better off forming the coalition structure $\pi_{K}$." That is: in the definition of the $\alpha$-core a contract among players cannot only specify the coalition, but also a partition of this coalition that the players form. Slightly abusing the terminology we can say that players have a larger action set in the cooperative game. 
There are two possibilities to correct for this. Either by restricting possibilities for deviation in the cooperative game or by expanding the action set in the sequential coalition formation game.

The first suggestion means that we rephrase the sentence above as: 'In the $\alpha$ ' definition, a group $K$ of players deviates if the members of $K$ are better off forming the coalition $K$.' In Example 1 this results $C \alpha^{\prime}(v)=C \alpha(v) \cup\{\{i, j, k, l\}\}$ so clearly $S E C S(v, \rho) \cap C \alpha^{\prime}(v) \neq \emptyset$, in fact, $\operatorname{SECS}(v, \rho) \subseteq C \alpha^{\prime}(v)$ can be shown generally. There are two problems with this approach. Firstly, it does not answer our second criticism, rather, it now also applies to the modified $\alpha$-core: it admits Paretodominated coalition structures. Dominance becomes so restricted, that even Paretodominance does not imply dominance. At the same time this restriction brings no benefits, so, for example, non-emptiness is still not guaranteed and therefore we reject this approach.

The second possibility is to modify the sequential coalition formation game: a proposal is now not only the coalition, but also the specification how this coalition is to be partitioned. So

$$
\sigma_{i}\left(h^{t}\right) \in \begin{cases}\{\text { Yes, No }\} & \text { if } \hat{\tau}\left(h^{t}\right) \neq \emptyset \\ \mathrm{T}\left(i, \hat{K}\left(h^{t}\right)\right) & \text { if } \hat{\tau}\left(h^{t}\right)=\emptyset\end{cases}
$$

where $\mathrm{T}\left(i, \hat{K}\left(h^{t}\right)\right)=\left\{\tau \in \Pi(T), T \subseteq N \backslash \hat{K}\left(h^{t}\right), i \in T\right\}$, the set of partitions that $i$ can form with the remaining set of players. ${ }^{1}$

As before, we are interested in the coalition structures that emerge as subgameperfect equilibria in stationary strategies, denoted by $\operatorname{SEC} S^{\prime}(v, \rho)$.

Firstly, we return to Example 1 and compute the $\operatorname{SECS}^{\prime}(v, \rho)$ for that game. For the two-player subgames the extended strategy set does not alter our arguments a great deal: proposing a singleton or proposing a partition into two singletons will lead to the same coalition structure, so either will be an equilibrium strategy. In the three-player subgames proposing a coalition of size three is still a dominated strategy. For other proposals we can say in general, that members of any proposed pair prefer to reject and propose a singleton: this will result in three singleton coalitions. This brings us to the root of the game tree. The first player will not propose coalitions of size 3 or 4 . By proposing a singleton it can get a payoff of $\frac{1}{18}$ and any player can get the same payoff by rejecting a proposal and proposing a singleton herself. Then a proposal will only be accepted if it offers at least this much to all players involved. This leaves two types of proposals on the table: proposing a partition of singletons (partitions of $1,2,3$, or 4 singletons) or a partition into two pairs. The latter is accepted by all, and it is also preferred by all, therefore were proposing singletons the strategy players would prefer to deviate and propose two pairs. Hence $S E C S^{\prime}(v, \rho)=\{\{i j, k l\}\}=C \alpha(v)$.

In general we have the following result:

Lemma 3. For any modified sequential coalition formation game $\Gamma^{\prime}(v, \rho)$ we have $\operatorname{SEC} S^{\prime}(v, \rho) \subseteq C \alpha(v)$.

Proof. In case $\operatorname{SEC} S^{\prime}(v, \rho)=\emptyset$ the result is trivial.

Otherwise consider $\pi \in S E C S^{\prime}(v, \rho)$. We show, by contradiction, that $\pi \in C \alpha(v)$.

\footnotetext{
${ }^{1}$ The proposal is really a coalition and one of its partitions, however from the latter the coalition can be derived and using only one of them simplifies our notation.
} 
Assume that $\pi \notin C \alpha(v)$. Then there exists a coalition $S$ such that for some partition $\pi_{S} \in \Pi(S)$ we have

$$
v_{i}\left(\pi_{S} \cup \pi_{N \backslash S}\right)>v_{i}(\pi) \text { for all } i \in S \text { and all partitions } \pi_{N \backslash S} \in \Pi(N \backslash S) .
$$

Now consider the corresponding deviation for the stationary perfect strategy profile $\sigma^{*}$ for which we have $\pi=\pi\left(\sigma^{*}\right)$. Consider a deviation by the member $i$ of $S$ who has the first chance to speak: if the set of players who have already left the game is $K$, then $K \cap S=\emptyset$. Suppose that this player rejects any ongoing proposal and suggests $\pi_{S}$ to form. As before, $\pi\left(\pi_{S}\right)$ denotes the coalition structure formed in case the proposal $\pi_{S}$ is accepted. We write $\pi\left(\pi_{S}\right)=\pi_{S} \cup \pi_{N \backslash S}^{*}$, where $\pi_{N \backslash S}^{*}$ denotes the partition that remaining players form following $\sigma^{*}$ together with the partition $\pi_{K}$ that has already formed. Then the payoff of player $i \in S$ would be $v_{i}\left(\pi_{S} \cup \pi_{N \backslash S}^{*}\right)>v_{i}(\pi)$ by Equation 3.2. Therefore the deviation is accepted by other players in $S$ and hence $\pi \notin \operatorname{SEC} S^{\prime}(v, \rho)$. Contradiction.

Hence $\pi \in C \alpha(v)$.

It is easy to verify that the definition of core stability introduced by Shenoy (1979) is equivalent to the following: The coalition structure $\pi$ is not core stable if there exists a coalition $S$ and partitions $\pi_{S} \in \Pi(S)$ and $\pi_{N \backslash S} \in \Pi(N \backslash S)$ such that

$$
v_{i}\left(\pi_{S} \cup \pi_{N \backslash S}\right)>v_{i}(\pi) \text { for all } i \in S .
$$

Therefore allowing players to propose partitions of coalitions does not alter core stability and $C C(v)=C C^{\prime}(v)$.

Proposition 4. Let $v$ be a valuation such that $C C(v) \neq \emptyset$ and for all restrictions $v^{\prime}$ of $v$ we also have $C C\left(v^{\prime}\right) \neq \emptyset$. Then for any rule of order $\rho$ we have $C C(v) \subseteq$ $\operatorname{SEC} S^{\prime}(v, \rho)$.

The proof of this proposition is analogous to the proof of Bloch (1996, Cor. 3.5). The following corollary gives a similar sufficiency condition to the one expressed by Bloch (1996, Lemma 3.4) on the nonemptiness of the set of stationary equilibrium coalition structures.

Corollary 5. Stationary equilibrium coalition structures exist if $C C(v) \neq \emptyset$ and for all restrictions $v^{\prime}$ of $v$ we also have $C C\left(v^{\prime}\right) \neq \emptyset$.

Corollary 6. Let $v$ be a valuation such that $C C(v) \neq \emptyset$ and for all restrictions $v^{\prime}$ of $v$ we also have $C C\left(v^{\prime}\right) \neq \emptyset$. Then for any rule of order $\rho$ we have

$$
C C(v) \subseteq S E C S^{\prime}(v, \rho) \subseteq C \alpha(v) .
$$

\section{RECURSIVE CORE}

Kóczy (2005) defines a pair of concepts that generalises the core to partition function games under the assumption that players are pessimistic/optimistic. Here we only use the pessimistic version (and hence drop the adjective in the sequel) and adapt it to the discrete partition function case.

First we introduce the notion of residual game:

Definition 4 (Residual Game). Let $(N, v)$ be a game. Let $S$ be a coalition and $R$ be its complement in $N$. Let $\pi_{S}$ be a multi-coalition deviation, a partition of $S$. 
Given the deviation $\pi_{S}$ the residual game $\left(R, v_{\pi_{S}}\right)$ is the discrete partition function form game over the player set $R$ and with the discrete partition function

$$
\begin{aligned}
v_{\pi_{S}}: \Pi(R) & \longrightarrow \mathbb{R}^{R} \\
\pi_{R} & \longmapsto v_{\pi_{S}}\left(\pi_{R}\right)=v\left(\pi_{R} \cup \pi_{S}\right)
\end{aligned}
$$

The residual game is a discrete partition function form game on its own and it can be solved independently of this deviation, or the initial game: The residual game is solved with the deviation taken as fixed, in fact independently of this deviation, or the initial game. We use this property to define the core:

Definition 5 (Recursive core). The definition consists of four steps.

(1) Trivial game. Let $(N, v)$ be a game. The core of a game with $N=\{1\}$ consists of the trivial partition: $C(\{1\}, v)=\{\{1\}\}$.

(2) Inductive assumption. Given the definition of the core $C(R, v)$ for every game with at most $k-1$ players we can define dominance for a game of $k$ players. Let $A(R, v)$ denote the assumption about the game $(R, v)$. If $C(R, v) \neq \varnothing$ then $A(R, v)=C(R, v)$, otherwise $A(R, v)=\Pi(R)$, the set of partitions.

(3) Dominance. The partition $\pi$ is dominated via the coalition $S$ forming partition $\pi_{S}$ if for all assumptions $\pi_{R} \in A\left(N \backslash S, v_{\pi_{s}}\right)$ we have $v\left(\pi_{S} \cup \pi_{R}\right)>_{S} v(\pi)$. The partition $\pi$ is dominated if it is dominated via a coalition.

(4) Core. The core of a game of $k$ players is the set of undominated partitions and we denote it by $C(N, v)$.

For a discussion about the various properties see Kóczy (2005).

\section{Sequential Coalition Formation and the Recursive Core}

The core is a static concept: once a core partition is attained, it is never abandoned. It does not, however, offer a recipe, or even a proof of the possibility to attain such a partition. In this section we establish the relationship between the core and the modified version of Bloch's noncooperative game of coalition formation.

5.1. Stationary equilibrium coalition structures. First we relax the sufficiency condition for the nonemptiness of the SECS'.

Proposition 7. Let $(N, v)$ be a discrete partition function form game such that $C\left(N \backslash S, v_{\pi_{S}}\right) \neq \varnothing$ for all residual games $\left(N \backslash S, v_{\pi_{S}}\right)$. Then $C(N, v) \subseteq S E C S^{\prime}(v, \rho)$ for all $\rho$.

Proof. The proof is inspired by that of Bloch (1996, Proposition 3.2) in part, and is by construction. We show that for a given $\tilde{\pi} \in C(N, v)$ there exists a stationary strategy profile $\tilde{\sigma}$ such that $\pi(\tilde{\sigma})=\tilde{\pi}$.

The strategy $\tilde{\sigma}$ is a function of the set $K$ of players who, at a certain point, have already left the game, their partition $\pi_{K}$ and the current proposal $\tau$ for a (sub)partition. Let also $\pi(\tau)$ denote the partition that the acceptance of a proposal $\tau$ ultimately produces. In the discrete partition function form game $\pi_{K}$, as a deviation defines a residual game $\left(N \backslash K, v_{\pi_{K}}\right)$. The "harsh response" to $\pi_{K}$ is an element $\tilde{\pi}_{N \backslash K}$ of the (by assumption non-empty) residual core $C\left(N \backslash K, v_{\pi_{K}}\right)$ ensuring that the deviation $\pi_{K}$ is not profitable. That is, $\tilde{\pi}_{N \backslash K}$ satisfies

$$
\begin{array}{ll}
\exists i \in S: & v_{i}\left(\pi_{K}, \tilde{\pi}_{N \backslash K}\right)<v_{i}(\tilde{\pi}), \text { or } \\
\forall i \in S: & v_{i}\left(\pi_{K}, \tilde{\pi}_{N \backslash K}\right)=v_{i}(\tilde{\pi}) .
\end{array}
$$


As $\tilde{\pi} \in C(N, v)$ such a $\tilde{\pi}_{N \backslash K}$ exists for all deviations $\pi_{K}$.

The stationary strategy $\tilde{\sigma}_{i}$ for player $i$ is the constructed as follows:

$$
\begin{aligned}
& \text { If } \pi_{K}=\varnothing, \quad \tilde{\sigma}_{i}\left(K, \pi_{K}, \varnothing\right)=\tilde{\pi} \\
& \tilde{\sigma}_{i}\left(K, \pi_{K}, \tilde{\pi}\right)=\text { Yes } \\
& \tilde{\sigma}_{i}\left(K, \pi_{K}, \tau\right)= \begin{cases}\text { Yes } & \text { if } v_{i}(\pi(\tau)) \geq v_{i}(\tilde{\pi}) \\
\text { No } & \text { otherwise. }\end{cases} \\
& \text { If } \pi_{K} \neq \varnothing, \quad \tilde{\sigma}_{i}\left(K, \pi_{K}, \varnothing\right)=\tilde{\pi}_{N \backslash K} \\
& \tilde{\sigma}_{i}\left(K, \pi_{K}, \tilde{\pi}_{N \backslash K}\right)=\text { Yes } \\
& \tilde{\sigma}_{i}\left(K, \pi_{K}, \tau\right)= \begin{cases}\text { Yes } & \text { if } v_{i}(\pi(\tau)) \geq v_{i}\left(\pi_{K}, \tilde{\pi}_{N \backslash K}\right) \\
\text { No } & \text { otherwise. }\end{cases}
\end{aligned}
$$

In equilibrium $\pi(\tilde{\sigma})=\tilde{\pi}$ and the strategy is stationary by construction so we only need to verify that it is subgame perfect.

We show this by induction. As subgame-perfectness holds for a trivial game we may assume that it holds for all games of size less than $|N|$.

Now consider game $(N, v)$ and observe the following. If a set of players $K$ have left the game to form $\pi_{K}$ the subgame is simply a coalition formation game with less players. Moreover, the proposed strategy exhibits the same similarity property: in equilibrium the core partition is proposed and accepted, while off-equilibrium residual cores are formed. The minimality condition then ensures that the offequilibrium path is subgame perfect so we can focus on Equation 5.3. We only need to check whether a deviation $\tau$ is ever accepted. This deviation corresponds to a deviation in the discrete partition function game. Since $\tilde{\pi} \in C(N, v)$ and by the construction of $\tilde{\pi}_{N \backslash K}$ we know that there exists a player $i$ in $S$ for whom the deviation $\tau$ is not profitable. Finally note that our strategy does not use a particular rule of order $\rho$.

This result, in combination with Lemma 3, enables us to provide both an upper and lower bound (in terms of set inclusion) on the modified stationary equilibrium coalition structures.

Corollary 8. Let $(N, v)$ be a discrete partition function form game such that $C(N \backslash$ $\left.S, v_{\pi_{S}}\right) \neq \varnothing$ for all residual games $\left(N \backslash S, v_{\pi_{S}}\right)$. Then

$$
C(N, v) \subseteq S E C S^{\prime}(v, \rho) \subseteq C \alpha(v) \text { for all } \rho .
$$

This result has the following consequence:

Corollary 9. Let $(N, v)$ be a discrete partition function form game such that $C(N, v) \neq \varnothing$ and $C\left(N \backslash S, v_{\pi_{S}}\right) \neq \varnothing$ for all residual games $\left(N \backslash S, v_{\pi_{S}}\right)$. Then for any rule of order $\rho, \operatorname{SEC} S^{\prime}(v, \rho) \neq \varnothing$.

As $C_{+}(N, v) \subseteq C(N, v)$ this corollary weakens the condition in Corollary 5 .

5.2. Order-independent equilibria. Following Moldovanu and Winter (1995, p.27) we define order-independent equilibria (OIE) and show that the order independent equilibria coincide with the recursive core.

Definition 6. A strategy profile $\sigma$ is an order-independent equilibrium for the sequential coalition formation game $(v, \rho)$ if it satisfies for any rule of order $\rho$ that 
(1) $\sigma$ is a stationary, subgame perfect equilibrium in $(v, \rho)$

(2) If $\sigma$ is played in $(v, \rho)$, the payoff vector $v(\pi(\sigma))$ is independent of $\rho$.

We denote the set of order-independent equilibria by $\operatorname{OIE}(N, v)$ and coalition structures (or partitions) resulting from playing such equilibrium strategies by $\operatorname{OIP}(N, v)$.

Theorem 10. Let $(N, v)$ be a discrete partition function form game such that $C\left(N \backslash S, v_{\pi_{S}}\right) \neq \varnothing$ for all residual games $\left(N \backslash S, v_{\pi_{S}}\right)$. Then $C(N, v)=\operatorname{OIP}(N, v)$.

Before proving this theorem we prove two auxiliary results.

Lemma 11. If Theorem 10 holds for all games with up to $k-1$ players, $\operatorname{OIP}(N, v) \subseteq$ $C(N, v)$ for all $k$-player games with nonempty residual cores.

Proof. The proof is based on the proof of Proposition A by Moldovanu and Winter (1995) and is by contradiction.

Assume that $\pi=\pi(\sigma) \in O I P(N, v)$, but $\pi \notin C(N, v)$. Then there exists a coalition $S$ such that a deviation $\pi_{S} \in \Pi(S)$ is profitable in the cooperative game for all assumptions about the residual game $\left(N \backslash S, v_{\pi_{S}}\right)$. In this game of nonempty residual cores this implies $v\left(\pi_{S} \cup \pi_{N \backslash S}\right)>_{S} v(\pi)$ for all $\pi_{N \backslash S} \in C\left(N \backslash S, v_{\pi_{S}}\right)$. In particular let $i \in S$ be such that $v_{i}\left(\pi_{S} \cup \pi_{N \backslash S}\right)>v_{i}(\pi)$. As $|N \backslash S|<k$, by Theorem 10, $C\left(N \backslash S, v_{\pi_{S}}\right)=\operatorname{OIP}\left(N \backslash S, v_{\pi_{S}}\right)$. The restriction of an OIE to a subgame is also an OIE, which, by our assumption belongs to the recursive core of the corresponding cooperative game. Therefore if deviation $\pi_{S}$ forms in the noncooperative game, the resulting coalition structure is $\pi\left(\pi_{S}\right)=\pi_{S} \cup \pi_{N \backslash S}$, where $\pi_{N \backslash S}$ belongs to $C\left(N \backslash S, v_{\pi_{S}}\right)=\operatorname{OIP}\left(N \backslash S, v_{\pi_{S}}\right)$. By our arguments for the cooperative game

$$
v\left(\pi\left(\pi_{S}\right)\right)>_{S} v(\pi)
$$

Without loss of generality let $\rho$ be such that $\rho(N)=i$. Consider strategy $\sigma_{i}^{\prime}$ for $i$ : "when no players have left the game, and it is $i$ 's turn to propose a partition, propose $\pi_{S}$ otherwise play $\sigma_{i}$." We show that this deviation from $\sigma_{i}$ is profitable for $i$ and hence $\pi(\sigma) \notin O I P(N, v)$, which is a contradiction.

To show this, consider another $j \in S$ and assume that after $j$ 's approval of $\pi_{S}$ the partition forms and $S$ leaves the game (either $j$ is the last player to accept or the rest is known to approve). We show that it is optimal for $j$ to approve. A rejection by $j$ makes her a proposer and the strategy profile $\left(\sigma_{i}^{\prime}, \sigma_{-i}\right)$ is played.

If, from here, no coalition ever forms, but the game goes on forever, the payoff for $j$ is 0 , which is clearly inferior as $0<v_{j}\left(\pi\left(\pi_{S}\right)\right)$. Then assume that coalition $T$ leaves the game first, forming partition $\pi_{T}$. Consider the part of the game from $j$ 's proposal until $T$ 's departure. If $i$ becomes the proposer once, as $j$ rejects his proposal he will be the proposer again and again: the game goes on forever without a coalition forming, contradicting our assumption that $\pi_{T}$ forms. Therefore $i$ is never a proposer. But then $i$ 's deviation is never played and playing $\left(\sigma_{i}^{\prime}, \sigma_{-i}\right)$ in $(v, \rho)$ is identical to playing $(\sigma)$ in $\left(v, \rho^{\prime}\right)$ with $\rho^{\prime}(N)=j$. By the assumption that $\sigma$ is an OIE playing it in $(v, \rho)$ or $\left(v, \rho^{\prime}\right)$ results in the same payoffs, which, by Inequality 5.6 are inferior to accepting proposal $\pi_{S}$.

Finally note that $i^{\prime} s$ deviation is limited to the game while all players participate. The game after the departure of a coalition is unaffected; in particular if $j \notin T, j$ 's payoff is unaffected by $i$ 's deviation giving the same conclusion. 
We have discussed all cases and found that $j$ 's refusal is never optimal. By backward induction the proposal is accepted by all players in $S$ and then, by Inequality $5.6 i$ benefits from the deviation.

Lemma 12. If Theorem 10 holds for all games with up to $k-1$ players, $\operatorname{OIP}(N, v) \supseteq$ $C(N, v)$ for all $k$-player games with nonempty residual cores.

Proof. The stationary-perfect equilibrium constructed in the proof of Proposition 7 is unconditional on any rule of order $\rho$. On the other hand, it produces the same coalition structure, $\tilde{\pi}$ for each rule of order $\rho$. It is therefore also an OIE. Such an OIE is constructed for each $\tilde{\pi}$ and therefore the result follows.

Proof of Theorem 10. The proof is by induction.

The result holds for trivial, single-player games.

Assuming that the result holds for all $k-1$ player games, the result for $k$-player games is a corollary of Lemmata $11 \& 12$.

\section{Conclusion}

Theorem 10 states that the core coincides with the order-independent equilibria of the sequential coalition formation game. This result is not so surprising considering that a similar relation has already been established for characteristic function form games without transferable utility (Moldovanu and Winter, 1995, Corollary 2.). Finally Huang and Sjöström (2006) show a similar result for their very similar r-core concept (Huang and Sjöström, 2003) with a modification of the continuous-time coalition formation process of Perry and Reny (1994). While orderindependence is part of the process by definition, establishing stationary subgameperfect equilibrium coalition structures is no easy task.

While these results bridge the gap between the cooperative and noncooperative approaches, one question remains, which is the relation of equilibrium strategies and equilibrium coalition structures. Here we have shown that coalition structures produced by order-independent equilibria coincide with the recursive core. Whether the same would hold for partitions that can be produced by equilibria for any rule of order, remains an open question we leave for future research.

\section{REFERENCES}

Bloch F (1995) Endogenous structures of association in oligopolies. RAND Journal of Economics 26(3):537-556

Bloch F (1996) Sequential formation of coalitions in games with externalities and fixed payoff division. Games and Economic Behavior 14(1):90-123

Chatterjee K, Dutta B, Ray D, Sengupta K (1993) A noncooperative theory of coalitional bargaining. Review of Economic Studies 60:463-477

Huang CY, Sjöström T (2003) Consistent solutions for cooperative games with externalities. Games and Economic Behavior 43:196-213

Huang CY, Sjöström T (2006) Implementation of the recursive core for partition function form games. Journal of Mathematical Economics 42(6):771-793

Kóczy LÁ (2005) A recursive core for partition function games. Mimeo.

Lagunoff RD (1994) A simple noncooperative core story. Games and Economic Behavior 7(1):54-61 
Lucas WF, Macelli JC (1978) Discrete partition function games. In: Ordeshook PC (ed.) Game theory and political science, New York University Press, New York pp. 191-213

Moldovanu B, Winter E (1995) Order independent equilibria. Games and Economic Behavior 9(1):21-34

Perry M, Reny PJ (1994) A noncooperative view of coalition formation and the core. Econometrica 62(4):795-817

Shenoy PP (1979) On coalition formation: A game-theoretical approach. International Journal of Game Theory 8(3):133-164

MAastricht UnIVERSiTy

E-mail address: 1.koczy@algec.unimaas.nl 